\title{
Discussion on Possibilities for Therapeutic Intervention in Children and Adults with Motor Disorders
}

\author{
Mijna Hadders-Algra ${ }^{1}$ and Albert Gramsbergen ${ }^{2}$ \\ ${ }^{1}$ Department of Neurology, ${ }^{2}$ Department of Medical Physiology, \\ University of Groningen, Groningen, the Netherlands
}

\section{INTRODUCTION}

\begin{abstract}
Albert Gramsbergen (Groningen, the Netherlands):
\end{abstract}
The contributions to this special issue of Neural Plasticity were written by the invited speakers to a workshop on Therapeutical Interventions in Motor Disorders which was held in Groningen, The Netherlands, from June 7 to 9, 2000. The aim of this workshop was to bring together scientists investigating fundamental aspects of movement control, as well as scientists and clinicians who are involved in therapeutical aspects of normal and disturbed neurological functioning. General aspects of movement control were discussed, as well as the normal and abnormal development of motor behavior and compensational processes after brain lesions. In addition, sections were devoted to pathophysiological aspects and perspectives for treatment of cerebral palsy (CP), Parkinson's disease, stroke and spinal cord lesions. Some challenges had to be met in this workshop. The hardest of these possibly was to bridge the knowledge which on the one hand stems from laboratories on movement sciences, from the neurophysiology of motor activities or the neuro-anatomy of descending pathways and on the other, the vast body of experience on the treatment of motor disorders obtained by clinicians and physiotherapists working in rehabilitation centers. Workshops as these often result in a collection of interesting contributions and at the best, the attendant or reader is able to select a few useful messages. Such workshops are only successful, however, when they lead to revisits or even changes in therapeutical strategies or when they initiate new experiments. That was our goal and as a further instrument to this, we scheduled at the end a general discussion to stimulate crossing the borders of disciplines. This discussion was recorded on tape and afterwards the discussants authorized the printed and edited contributions. Because several intriguing and sometimes clarifying points were raised, indicating that bridges had been made, we decided to include this round table discussion at the end of this special issue. The aim of this last paper is to highlight the problems and the issues which are at stake in the field.

\section{STEM CELLS}

Mijna Hadders-Algra (Groningen, the Netherlands):

There is quite a gap between knowledge and experience from the basic neurosciences and what is happening in the clinic and one of the questions which did not come across clearly is the problem what can be done with the results from research in the basis sciences such as new findings on stem cell application in cases of neural injury at young or adult age. Therefore I would like to ask $\mathrm{Dr}$ 
Leenders what the perspectives are in this field of research for future treatment. Will treatment with stem cells be limited to treatment in Parkinson's disease or could this type of therapy in the future also form a mediator of recovery after brain lesions at early age?

Nico Leenders (Groningen, the Netherlands):

The use of stem cells, indeed, seems very promising and potentially the applications might be enormous. Yet, the research field itself is by no means ready to predict what the therapeutical benefits might be. It looks now as if diseases with focal lesions in the brain, that is, lesions in which a limited number of neural cells is damaged, might be successfully treated with stem cell implantations. During the last 10 to 15 years, it has been demonstrated repeatedly that cells which are processed adequately in the laboratory can survive in a host, where they can take over functions, grow axons, and make functional connections, both in a pharmacological and clinical sense. The therapeutical effects all depend, however, on how many cells can be stimulated to survive. This is a general cell-biological problem on which many laboratories are working at the moment. Implantations of stem cells seem particularly promising for successful therapy when the basic pathological problem is a circumscript lesion, such as is the case in Parkinson's disease and Huntington's disease, and there may be a few others. But when you are talking about more diffuse pathological conditions such as Alzheimer's disease, a post-stroke condition, or epilepsy, I am hesitant to predict successful therapeutical applications in the near future.

Nowadays, the research in the area of stemcell breeding is concerned with questions as how easy it is to produce specific cell types with specific capacities, and how their survival in adequate numbers can be accomplished. When these problems are solved, the next and even more complicated problem is how relevant is the replacement of lost neurons to the nervous system in specific pathological conditions and how well does it serve the patient. Considering stem cell treatment in cases of CP or epilepsy: I think that these disorders are too heterogeneous in terms of the site of the lesion and the type of the cells involved, for stem cell therapy becoming a realistic mode of intervention.

Albert Gramsbergen (Groningen, the Netherlands):

There are important differences in the regrowth of axons after lesions in the central nervous system and those occurring after transections of nerves in the peripheral nervous system (see Gramsbergen, this issue). The differences seem to depend on the difference in properties of the oligodendroglial cells in the central nervous system and those of the Schwann cells in the peripheral nervous system. The Schwann cells seem to stimulate the outgrowth and maintenance of nerve fibres by the production of neurotrophic factors while oligodendroglial cells in the central nervous system seem to inhibit out-growth and possibly even the differentiation of unproliferated cells. Could you comment on this Dr Kolb?

\section{Bryan Kolb (Lethbridge, Canada):}

There clearly is a fundamental difference between glial cells in the peripheral nervous system and the central nervous system, and this applies to oligodendroglial cells and Schwann cells, as you mentioned. We demonstrated that astrocytes in the central nervous system can be advantageous after lesions as they can be induced to produce trophic factors which may stimulate neurogenesis. There is as yet no evidence that this also happens in the peripheral nervous system. But, as we do know that the stem cells for some 
peripheral nerves remain lying along the ventricular zone in the spinal cord, neurogenesis occurring after a lesion of the peripheral nervous system remains a theoretical possibility.

Coming back to the issue of stem cells, in my talk (see Kolb, this issue) I addressed the possibility that rather than implanting stem cells we might pursue the possibility to have the brain generate its own endogenous stem cells in order to solve the problems after injury. We demonstrated that in young animals, this proliferation may occur spontaneously. In addition, we also showed that it is possible to infuse trophic factors in adult animals, which in animals with stroke resulted in the generation of thousands of progenitor cells capable of producing both neurons and glial cells. In case of focal injury, the newly generated cells were shown to migrate to appropriate locations. Nothing in principle seems to prevent the newborn cells from turning into fully differentiated neurons, but as I pointed out previously the neurons in our experiments did not differentiate. This means that we probably are missing the relevant differentiation signal. If we were able to identify this signal, we might be able to treat focal lesions of the brain by getting the brain to do the repair process itself rather than by implanting foreign stem cells into the lesion. I agree with Dr. Leenders that similar types of treatment might not be realistic for disorders with a less localized dysfunction of the nervous system, such as Alzheimer's disease or epilepsy.

\section{EFFECT OF PROPRIOCEPTIVE INPUT}

\section{Margaret Yekutiel (Hofit, Israel):}

Dr. Scrutton presented data on gradually developing deformities in children with $\mathrm{CP}$, such as very flexed positions of the wrists and dis- location of the hips (cf., Scrutton \& Baird, 1997). I wondered if the children he showed us were not able to achieve a functional position of the wrist or hips because of a sensory deficit. Crothers, Tizard and Paine back in the fifties (Tizard et al., 1954; Crothers \& Paine, 1959) studied a long list of patients with CP whom they had seen over 30 years. When they called them up for re-examination, they found that about $60 \%$ of the patients, most of them being persons with spastic hemiplegia and some of them being persons with spastic diplegia, had a sensory loss, deficits in proprioception, and other types of sensory dysfunction, which they had not noticed before. They suggested that the sensory deficits might be the explanation for the relatively poor results of surgery in the treatment of deformities. Dr. Scrutton said that he could not find a factor which distinguished between successful and unsuccessful intervention in the treatment of developing deformities. I wondered whether an absence of sensory feedback might be this factor.

\section{David Scrutton (London, United Kingdom):}

Because I could not identify this factor, I cannot answer your question, but you may well be right. Obviously, many of the group of children and adolescents I discussed had no experience of hand use. Presumably, and in line with what we have been hearing during this workshop, a factor which might play a role also is a centrally originating disuse. However, I do not have the faintest notion whether this could be a factor in the children I discussed. The studies you mentioned did not report a failure to correct the deformity (it was surgical correction), but a subsequent failure to use the uncorrected and corrected hand/wrist. The problem I was discussing was, that by conservative means, some can be corrected and some cannot; and 1 cannot see the difference prior to treatment. 


\section{MOTOR MEMORY}

Aleid van Wassenaar (Amsterdam, The Netherlands):

I was fascinated by the holistic concept mentioned by Dr. Mulder and the use of memory in getting people to walk again. But being a pediatrician and working with infants in whom the brain is damaged at very early age, I wondered how this memory concept could be applied in newborns, who might have a memory of general movements in utero (see Hadders-Algra, this issue). I wonder whether Dr. Mulder or Dr. Hadders-Algra might comment on this.

Mijna Hadders-Algra (Groningen, The Netherlands):

Dr. Mulder was mentioning the impact of memory in reestablishing movement patterns such as walking. However, we know little about the development of motor memory in early life. Simple, implicit memory operations like habituation develop during fetal life, but more complex, explicit, and conscious memory functions first emerge at the end of the first postnatal year (Hepper, 1997, Rovee-Collier, 1997). Thus, it seems unlikely that motor memory in young infants can be used as a mediator of improving the quality of general movements after brain damage. Rather, I think that, at early age, the tricks to improve movement quality might be to motivate infants to move and to explore the large range of movement possibilities potentially available. So, basically, I think, what you should do in young infants with motor disorders is to play with them in many different situations to exploit these aspects.

Theo Mulder (Groningen, the Netherlands):

When children grow older they of course have formed a memory of movements. For instance, I have been told that in comatose children, the voices of relatives or parents sometimes are used as a stimulus. The idea here is that the voices are recognized on the basis of memories from the precoma period. In a similar vein, you could use the same strategy in children who acquired a brain lesion at older age to reestablish movement patterns which they had used before the injury occurred. But, I agree with Dr Hadders-Algra, such therapies probably will not apply to newborns as they lack the storage of pre-morbid memories.

Mijna Hadders-Algra (Groningen, The Netherlands):

Indeed, memory might not be the most appropriate way to stimulate movements in young infants. But, another aspect Dr. Mulder mentioned could perhaps be used, namely the tendency of young infants to imitate movements. Again, the playful situation would offer the setting in which you could show the infant how to move and how to solve a motor problem.

Theo Mulder (Groningen, The Netherlands):

Wasn't it Melzoff who showed that newborn babies were able to imitate facial movements?

Mijna Hadders-Algra (Groningen, The Netherlands):

Yes, indeed, it was Melzoff. Meltzoff and Moore (1977) reported that newborn babies especially could imitate mouth opening and tongue protrusion movements of adults. However, a recent review indicated that the imitational capacity of newborns was restricted to tongue protrusion movements only (Anisfeld, 1991). Moreover, the elegant study of Jones (1996) indicated that the tongue protrusion behavior was not based on imitation but on joint activity. When babies get excited they produce a lot of exploratory tongue 
movements, both intra- and extra-orally. Seeing an adult with a protruded tongue appears to be a very exciting experience for young infants, at which they react with tongue movements, including tongue protrusion.

\section{Roberta Shepherd (Sydney, Australia):}

Just a brief point that may relate to memory. My clinical colleagues train very young (as early as 6 to 8 weeks) infants with developmental delay/ cerebral palsy in the actions of standing up and sitting down and also standing up from crouch. We think this utilizes an innate flexion and extension of hips, knees, and ankles which occurs in utero and is seen at birth if the infant is placed with feet on floor. Ability to perform this motor pattern (raising and lowering the body mass over fixed feet) is critical to the development of many mature motor actions. Does this relate in some way to memory or is it solely evidence of the emergence of movement out of the biomechanical possibilities inherent in the musculoskeletal linkage?

\section{Mijna Hadders-Algra (Groningen, The Netherlands):}

I would like to comment on that. The problem here is what you call memory. Two main types of memory can be distinguished, conscious (explicit) and unconscious (implicit) memory. The memory Dr. Mulder was talking about (see Mulder \& Hochstenbach, this issue) is the conscious type of memory, and I doubt whether a conscious memory exists for movements in the prenatal period.

\section{Albert Gramsbergen (Groningen, the Netherlands):}

We are talking about memory, motivation and emotion and the brain is involved in many of these processes. What I would like to ask Dr Lemon is, when we are talking about these central mechanisms in newborn infants we should realize that not all connections which are present in the adult have been established yet. You are an expert on the development of descending projections. Could you comment on how emotion could influence movements in the neonatal period, how memory could be established at early stages of development and how movements which are perceived by the visual system could be imitated by the young child. Are the motor systems ready to be modulated by these processes or to process the information?

\section{Roger Lemon (London, United Kingdom):}

One has to be aware that although the brain is able to rehearse certain programs, at all times it is constrained by what it has at its disposal in terms of internal connections and also by constraints of the skeletomuscular system (Lemon et al., 1997). Clearly there are massive changes, both prenatally and postnatally, in the human motor system and therefore whatever is happening in terms of central motor programs, this must keep in step with all these changes. For instance, conduction velocities become very much faster, muscles become stronger, bones become longer, to mention only a few aspects of the huge number of changes which are going on. The brain needs information about all these changes in order to tailor its commands to be appropriate.

Janet Eyre and her group in Newcastle have been suggesting that the human corticospinal system develops according to an earlier timetable than in nonhuman primates (Eyre et al., 2000); they suggested that this system plays an important role in controlling the maturation of the spinal motor machinery. 


\section{THE BRAIN, A SELF ORGANIZING SYSTEM?}

\section{Roger Lemon (London, United Kingdom):}

When talking on brain lesions and motor performance, I think there is a more general issue here. It seems to me that there are important behavioral outcomes of lesions that occur at particular times and which affect particular structures (cf. the contributions of Gramsbergen, Hadders-Algra, Kolb, Mayston, this issue). I think, that a system which is purely self-organizing, as some investigators claim it to be, would not be as sensitive to damage as the human brain is. I am reinforced in my view by realizing that in fact, the capacity of the brain to repair itself is very limited. There are fundamental mechanisms within the central nervous system which limit such repair, and one possible reason for this is that repair of significant damage to such a complex structure is just not feasible. Like most people, I am impressed and excited by the work referred to by Dr Mulder on the capacity of the brain for self-organization and the fact that maps within the brain are updated continuously on the basis of experience. However, one should not lose sight of the fact that a large fraction of our genome is concerned with the specificity of connections within our brain, connections that allow the developing brain to deal with and organize activity within our sensory, motor and other systems. If you don't have an appropriately 'wired' brain (and I do realize that hard-wiring is completely out of fashion in these days), you may have serious problems in making any use of the barrage of incoming information. For example, when you move your knee, about 10 million impulses are generated per second. I do not believe that a system that is not appropriately wired would be able to cope with that amount of information. This specificity may help us to explain why some brain lesions have such devastating effects.

Bert Otten (Groningen, the Netherlands):

I agree with that. Some 10 years ago, experiments were going on with robots that had to manipulate objects, turning them around and put them on a table. In order to be able to perform these tasks, the robots needed to have some description of their own body, such as dimensions and inertias. When such descriptions were lacking the robots were never able to reach the right solutions. In other words, a reasonable set of prior knowledge is a prerequisite for motor learning. With respect to plasticity, which is very much en vogue nowadays, I would say that a hard wired system, or a hard wired brain if you wish, is the essential starting point setting the boundaries for plasticity.

\section{EXPERIENCE}

Roberta Shepherd (Sydney, Australia):

I would like to ask then: How important is experience and activity and to what extent do they affect neural connectivity? If experience and activity (and therefore exercise and training in rehabilitation) affect connectivity in any way, this means that what happens in rehabilitation is very important. This would be equally true for babies making connections or in adults after brain lesion or following cellular implants in the brain in some future time. It is important to test how infants or adults might benefit from different experiences and training methods in terms of neural reorganization in order to determine which rehabilitation 
methods have positive and which have negative effects.

\section{Roger Lemon (London, United Kingdom):}

There is a struggle going on in early development, an incredible competition. Every neuron is fighting for survival, as in the developing brain up to $50 \%$ of the cells are going to die in a competitive, almost 'Darwinian' fight for survival. An important factor in deciding the outcome of that struggle is experience-related neuronal activity. To give you an example, at early stages the corticospinal system in cats projects bilaterally. In other words, each side of the brain talks to both sides of the spinal cord. Whether or not the system remains bilateral depends on the interaction between the two hemispheres. During normal development the ipsilateral projection is withdrawn, as basically the active projections from the contralateral hemisphere exclude the ipsilateral projections from 'their' half of the spinal territory. When you inactivate (not lesion) the contralateral cortex by keeping it suppressed by a GABA agonist, this allows the ipsilateral projection from the other hemisphere to be sustained (Martin et al., 1999). So, clearly activity is what is needed to maintain representations. The issue is-how did the representations become established in the first place? I do not believe that these primary representations are established simply on the basis of experience. The initial maps are laid down globally and experience plays an important role in shaping the ultimate wiring. It is much as $\mathrm{Dr}$ Hadders-Algra showed us-in her extrapolation of Edelman's ideas on neuronal group selection to human motor development-that within the boundaries of globally predefined populations of neurons a dynamic organization occurs, whereby particular groups of neurons become organized for particular functions (see Hadders-Algra, this issue).
Essential to Edelman's thesis (Edelman, 1989) is that the neurons belonging to neuronal groups should be close together. There are very good reasons why neurons which do the same things, need to be close together. The main reason is that close distance is ergonomically efficient to organize the connections between the cells with a minimum amount of space occupied. Space is a big feature in how brains are designed; the brain cannot afford itself to use up the entire space just to wire things together. So there is a key interaction between the organization you start off with and the experience you then acquire through the working operation of that circuitry.

\section{Margaret Mayston (London, United Kingdom):}

Thinking about the problem of experience and activity, I think the organism also has to make some sense out of the activity and particular experiences. Here we maybe face one of the limitations for some of the children with $\mathrm{CP}$ who do not have the cognitive abilities to really make use of the experiences that they have. These children may be able to learn some kind of movement but they cannot use it in the right context, because they cannot understand what the movement should do for them. Therefore, cognitive ability is another point to keep in mind when discussing the effects of experience and activity on motor behavior in subjects with a lesion of the brain. Because I think that cognition and motor experiences are elements that drive each other.

\section{Bryan Kolb (Lethbridge, Canada):}

One of the consistent findings in rats who had a lesion of the brain at early age is the presence of various types of peculiar connections which probably interfere with normal function. These connections are there, because they did not die. In 
other words, these connections also are present in normal animals at early stages of development. But normally they disappear, presumably on the basis of experience. Perhaps one of the goals of therapies with increased experience in infants who acquired a brain lesion at early age might be to get rid of these peculiar, retained connections. One of our working hypotheses is that getting rid of these peculiar connections rather than producing new ones is what early interventions actually do.

Theo Mulder (Groningen, the Netherlands):

I would like to clarify my point of view. $\mathrm{Dr}$ Lemon was talking about the 'hard wired' properties of the system as a prerequisite for information processing. I agree that the system is hard wired but that is not identical to saying that the system is rigid. The activity of the networks can be modulated by activation or stimulation and that implies that the system is everything but rigid or static.

\section{Roger Lemon (London, United Kingdom):}

You are right. In this respect it is relevant to refer to the work of Merzenich. Merzenich and colleagues did some fascinating experiments (Buonomano \& Merzenich, 1998). The basic issue that their studies sought to tackle was how does the cortex 'know' the difference between two digits? Merzenich's answer would be that the cortex largely identifies the digits by the temporal profile of activity. They did a nice experiment in which they found that different types of experience lead to reorganization in the somatosensory cortex. If tactile stimuli were repeatedly passed across two digits - that is, in a transverse direction - then the cortical 'map' became reorganized in such a way that the transversely neighboring finger areas were represented as a single finger. So, it is the organization of the connections which allow the temporal evolution of a sensory stimulus to be interpreted and organized by the cortex. One could argue that a finger does not have any real meaning to the cortex, it is simply a map of space and time.

Charlotte Häger-Ross (Umeå, Sweden):

I just would like to return to the fantasies and the visionary world, eluded to by Dr Mulder. Imagine that the Society of Movement Disorders would provide a huge grant for intervention projects and that you would be on the board of this society to decide which type of projects on intervention should be given priority. How would your reasoning be for awarding projects and what would be your criteria for selection?

\section{Theo Mulder (Groningen, the Netherlands):}

This is difficult to answer. If I had the power to do that, I would stimulate the projects which had a sound theoretical basis and in which the applicants had the courage to be unorthodox. I do not know whether Ramachandran ever has asked for funding for his work on phantom limbs, and if so, whether he would have got the money for his project on the use of mirrors to study sensation in phantom limbs. The idea of the mirror was that the mirror projected motor behavior of the intact limb to the site of the phantom limb and to ask the subject about sensory experiences. With or without grants, Ramachandran did the experiments and he was successful (Ramachandran \& Blakeslee, 1998). So I really would stimulate those experiments which could help us to come any further rather than finance mainstream work. We only are able to make progress when we dare to go into novel, be it risky directions, when we invest in multidisciplinary research and have the courage to look over the boundaries of our own field of research. 


\section{EFFECTS AND LIMITATIONS OF TREATMENT}

\section{David Scrutton (London, United Kingdom):}

I can not tell anybody how to achieve effective treatment in patients with neurological disorders, but at the sharp end it would be very helpful to know at the earliest possible time what is impossible for us to achieve. So that we can concentrate our aims in other directions. Now we waste an awful lot of time finding out that something cannot be achieved, and even then cannot be certain whether it is the extent of the disorder, locomotor development or the choice of treatment which is the problem. So it would be tremendously helpful to know what the limits of achievement will be in patients with specific types of lesions of the brain.

\section{Tatjana Velickovic (Ljubljana, Slovenia):}

I do not agree with this pessimistic approach. In habilitation procedures we really never take the lesion as our starting point but instead we try to make use of the potentials of the unaffected parts of the brain.

David Scrutton (London, United Kingdom):

I was asking for a truthful and real answer on the question in which cases any improvement would not actually apply. In children with a lesion of the brain, and especially in severely handicapped children, we always run to a point of failure with our treatments, a point we cannot get beyond. I would like to know what that point is before I start with treatment, because the rest is wishful thinking.

Riclef Schomerus (Dortmund, Germany):

I want to comment on what you said with regard to this endpoint of a treatment. I wonder whether such a point ever exists because new approaches in treatment are always being developed. Recently more functional approaches have been developed, which resulted for some patients in improvements which previously had not been expected. Thus, to my opinion you never can be sure that there is an endpoint in therapy.

David Scrutton (London, United Kingdom):

You all are going around what I actually said. What I want to know is what we cannot achieve. What you are talking about is what we might achieve. That is the difference. I'll give it another try to make myself more clear. We have to agree that if a child has got $\mathrm{CP}$, he/she never will be going to function normally. If you don't believe that, then we are talking in two different worlds! What I am asking for, is that as soon as possible, which may be at 1 year old, may be at 4 years old, or even perhaps at the age of 25 years, but as soon as somebody can tell me that something is impossible for that child, I want to know it. I want to know it because I don't want to waste my time and-much more importantly-I don't want to waste theirs. That is what I am asking for.

\section{ADAPTATION AND PLASTICITY}

Mijna Hadders-Algra (Groningen, The Netherlands):

One of the issues during this conference was, and I think Dr Lemon brought it up in his lecture, is the question whether adaptation and plasticity are two separate processes or whether both are aspects of a continuum. If there is a difference between the two of them, I would like to know what is happening in, for instance, CP. In the 
section on CP (see Mayston, this issue) we heard about the dysfunctions which are present in CP. Should those changes be regarded as adaptive changes or as plastic changes? If they are adaptive changes, are they a just a good solution or, on the contrary, should they be treated (see also Shepherd, this issue)? Could Dr Mayston comment on this?

\section{Margaret Mayston (London, United Kingdom):}

This is an ongoing and interesting discussion. I said the other day in relation to Dr Lemon's contribution (not published in this issue) that the nervous system has many ways to achieve the same objective. Maybe children with CP lose some of the varieties of ways of achieving the same objective, so they become more limited in their choices. And yet, if we look at the young child who is not severely involved, we see that over time the child often loses some of the choices he or she started of with. This then poses the question, how much can we retain of what these children have when they are younger. Moreover, we should investigate why they lose some of their possibilities. Is it because we don't give them the right experiences? Is our therapy too passive? We worry about stretching the muscles and decreasing tone, but instead, we should perhaps stimulate the children and make them more active. Coming back what you said earlier about playing with the child, I think therapists should be taught to play with the children rather than to do exercises with them. Because young children cannot do exercises, but they do want to play. Maybe we should motivate children in their play, so they can explore their potential. This would hold true for the children who seem to have the potential to improve their motor function. For the more severely impaired child, perhaps our goal lies more in better management.

\section{Roberta Shepherd (Sydney, Australia):}

I think that the most important outcome in rehabilitation is the achievement of effective and efficient motor behavior. Therefore I have a simple view of adaptation. An adaptation is negative ('bad') if it prevents effective and efficient performance of whatever the person wants to do, and it is positive ('good') if it enables him or her to perform the movement effectively (in terms of goal attainment) and efficiently (in terms of cardiovascular and muscular output). This simplification is a convenient point to start from.

\section{Margaret Mayston (London, United Kingdom):}

But what to do in the following situation? You have a child who achieves the desired objective, for instance to grasp the toy, but it does it in a qualitatively poor way-for instance solely by rolling over with a lot of flexion. If the child would continue to do so, it could develop contractures.

Roberta Shepherd (Sydney, Australia):

It's not so important that the action is 'qualitatively poor'. Rather it is important that the infant develops flexible motor performance-that is, that he or she can grasp objects in sitting or standing; that she or he can grasp objects and use them for different outcomes. If the infant is only on the floor during therapy, then the opportunity to practice and learn these actions in a flexible manner is not provided. You might put the child in a chair or you might support the child in a brace in standing, where he or she would not be able to make rolling movements, and use training methods that promote effective muscle activations in a range of different contexts. The environment can be used in such a way that the required motor behavior is 'forced'. There are many ways of 
providing constraints which 'force' more effective muscle activations, as in Taub and colleagues' studies with adults (Taub et al., 1993).

\section{Milivoj Velickovic (Ljubljana, Slovenia):}

I just wish to stress that it is important to specify the age of the patient. It clearly makes a great difference for therapy whether we are talking about newborn infants, school-age children or adult patients. I think it is important that we differentiate between 'early brain damage' and 'cerebral palsy'. Because in case of early brain damage - that is, the occurrence of a lesion of the brain at an early stage of development-we have the opportunity to exploit by means of early physiotherapeutical intervention the plastic and compensational capacity of the young brain. So, probably we can gain something in terms of really improved motor behavior at early age-that is, before the age of three months. When children have developed a clear clinical picture of $\mathrm{CP}$-that is, when the pathology is settled, we mainly can help the children with symptomatic therapies like stretching, surgery and pharmacological treatment.

\section{Margaret Mayston (London, United Kingdom):}

It should be realized that it is still unclear whether we can achieve an improvement of motor performance in children with developmental motor disorders by means of early intervention. For instance, the Liverpool study on early intervention in at risk infants was unable to demonstrate a difference in developmental outcome between infants who obtained intervention and those who did not (Weindling, 2000).

Nico Leenders (Groningen, the Netherlands):

Coming back to the issue of adaptation, plasticity, flexibility, changes in connectivity and the like, my view is that the brain is extremely rigid. I know, it is very much en vogue to talk about adaptation and plasticity. Adaptation certainly exists, but about plasticity I am less sure, it probably is more an exception than a rule. What strikes me, is that the brain is so remarkably stable and that it has such a rigid composition and yet enables the expression of many complex and changing functions. Only on the basis of the brain's 'stability' we can process information in a predictive way and 'function'. I am fascinated by the paradox that movements, which can be produced in so many different ways due to the numerous degrees of freedom offered by our muscles and joints, require a system consisting of brain centers in relatively fixed positions with relatively determined connections, a system which is laid out in the blue print of our genes. So, at a basic level, I think the plastic capacity of the brain is limited indeed. This view is supported by common clinical knowledge that it is very difficult to treat adult patients with movement disorders.

Bert Otten (Groningen, the Netherlands):

The fact that Dr Leenders is able to say that shows how little we know. On the basis of hard and objective knowledge it is impossible to contradict him. Still, I think there are many observations that indicate that he is wrong. For instance, rapid reorganisation of the sensory cortex after peripheral trauma just shows how plastic the nervous system can be. Now, this is plasticity inside neuron pools. Little knowledge exists on rerouting between functionally different centres.

Milivoj Velickovic (Ljubljana, Slovenia):

Once again I would like to stress that the brain of the newborn infant differs largely from that of 
an adult. The child is not a small adult and consequently it is impossible to compare the plastic capacities of a young and an adult nervous system.

Bryan Kolb (Lethbridge, Canada):

Age is related to the amount of experience an individual has and experience is an important element in the effects of intervention. One of the things which we have seen in our experiments is that experience given to the animals before the lesion occurred could affect the way in which recovery took place. Also interventions which were given after the injury but before any behavioral symptoms emerged-as the animal on the basis of developmental processes in the brain could not yet produce the specific motor behaviorcould affect the outcome. So it is clear that early intervention-even in the absence of knowledge on the nature and effects of the injury-might make a difference in outcome. The obvious question then is 'Which specific interventions should be given at what age in clinical situations?', but there we are back to ignorance.

\section{REPRESENTATIONS OF MOVEMENTS: POSSIBLE ROLE OF IMAGINATION IN MOTOR REHABILITATION.}

\section{Anand Nene (Enschede, the Netherlands):}

I have a question to Dr Otten and Dr Mulder. I was quite fascinated with the theory on imagination playing a role in maintaining orderly representations or mapping. But Dr Otten just told us that such representational maps may change quite rapidly after an injury occurs. So now I wonder where does this leave us with the imaginary maps?

Bert Otten (Groningen, the Netherlands):

It is obvious from a number of studies that the planning of a movement involves the activation of brain centers, and this also occurs when the movement is hindered or cancelled at the latest moment. So, when you acquired a lesion or when your arm is amputated, you still may be able to plan the movement without the actual movement taking place. This is what I implied with imagining.

Theo Mulder (Groningen, the Netherlands):

I also think that there could be several levels of representation. For instance, it could be surmised that the fast changes which take place as a result of afferent disinformation take place at another level of representation than the representation as a result of imagination or of conscious reflection on movements. So, there is a multitude of representations and a multitude of levels of control. Other examples are the famous cases of patients with a total amnesia described by Joseph LeDoux (1996) and Brenda Milner (Milner et al., 1968). Typically for these patients is that they forget the person with whom they had a conversation as soon as the person has left the room. To test memory functions of a woman with total amnesia, a doctor shook hands with her at the end of a consultation, but the trick this time was that he had a pin in his hand, which turned the handshaking into a painful event for the patient. The doctor left the room and returned after five minutes. Like before, the woman did not recognize the doctor, but she all of a sudden was very reluctant to give him a hand. So, there are different levels of representation in the nervous system which normally continuously interact. 


\section{Bert Otten (Groningen, the Netherlands):}

I agree with this point. In this respect it is illustrative that in a lot of automatic movements thinking about the actual movement should be avoided as it interferes with movement performance. For instance, when you are in a fast curve during speed skating and simultaneously analyze the movement consciously, this usually results in falling. This probably means that as soon as the motion has been automatized it is processed at another level in the CNS than at the conscious level.

Nico Leenders (Groningen, the Netherlands):

I have a comment on the use of the word 'mapping' in the discussion. The word mapping might be confusing in this context and this holds also true for the word 'representation'. If you imagine something or do something, this leads to changes in the perfusion of specific parts of the brain as visualized by fMRI or PET. This indicates that these particular parts of the brain in the one or in the other way are involved in the process investigated. However, it is unrealistic to think that the areas with visible changes in perfusion (the 'maps' or 'representations') during imagination or moving are the only areas involved in the representation of that function. In other words, the terms mapping and representation of a particular function can be very misleading.

\section{Roger Lemon (London, United Kingdom):}

I would like to mention another aspect of the issue of what the different systems actually are doing. If you look at the comparative anatomy of the corticospinal tract in lower and higher mammals (Porter \& Lemon, 1993; Lemon et al., 1997), it is interesting to see that in rats, the descending fibres largely project into the more dorsal parts of the spinal grey matter (that is, the dorsal horn), indicating that the corticospinal tract in these animals is involved in controlling, gating, and filtering sensory input. In animals with more advanced limb function, the corticospinal tract becomes additionally involved in the control of reflexes and then ultimately, in many primates, also in the direct control of motoneurons. I often wonder whether some of the problems which we see in children with $\mathrm{CP}$ and in adult patients with stroke is not so much an inability to move but rather a malfunction of the descending control system that deals with the sensory input, or reafference, that results from the movement. Because, if you are not able to deal with that information, one of the simplest solutions is to just stop moving. Therefore, I think the idea of learned non-use is such a powerful concept (e.g., Wolf et al., 1989). If you cannot analyze the information from a part of body or if the information gives rise to pain, it makes extremely good sense to stop moving it.

\section{ONSET OF THERAPY, DESIGN OF EFFECT STUDIES}

Anand Nene (Enschede, the Netherlands):

Another point is what the relation should be between the time of injury and the onset of the imagination therapy as proposed by Dr Mulder in order to obtain an effect (see Mulder \& Hochstenbach, this issue).

Theo Mulder (Groningen, the Netherlands):

I think that you should start with the therapy as soon as possible, because we know that neural decay after brain injury is a relatively fast process 
and the hypothesis is that this decay might be inhibited by imaginary movement therapy. I am not quite sure about the effects of these types of early treatment because nobody did these experiments in the context of rehabilitation and health care.

\section{Alexander Geurts (Nijmegen, the Netherlands):}

We already for a long time speculated about intervention therapy and the processes which are involved. I think we already can draw one main conclusion and that is that we need well controlled studies in well selected groups of patients to see what is the real effect of specific interventions. This in line with a remark made by Dr. Scrutton the other day stating that we should not investigate long-term treatments which are vaguely defined. Rather we should put our efforts in the investigation of concrete and well defined interventions.

\section{Roberta Shepherd (Sydney, Australia):}

I think it is interesting what you are saying. I wish that journal editors would not publish articles on outcome studies which only mention that patients had 'physiotherapy for two weeks' without any further specification. We must get to the point where we give details of exercises, training etc., with dosages (e.g., number of repetitions). Only in such a way can we determine the effects of particular treatments. There are still too many studies published with ill defined interventions, which means that we do not know what the results actually mean. The kind of very discrete study you suggest might also include testing of the biomechanics (that is, performance) of movement, testing of the patient's satisfaction with their own performance in their own environment, and some form of neuroimaging or neurophysiological testing which would give some indication of the neural processes and biomechanical effects of intervention.

\section{FINAL REMARKS}

Mijna Hadders-Algra (Groningen, The Netherlands):

In line with good tradition in science our meeting on therapeutical interventions in motor disorders generated more questions than answers. Questions which we should address in the near future, such as:

- What are the pathophysiological mechanisms of the various types of motor disorders? Answers to this question will form the vital basis for the development of effective intervention therapies.

- What can be considered as a functional and what as a dysfunctional adaptation after a lesion of the nervous system? Knowledge in this area could result in a shift of therapeutical goals, for instance, it could result in a change in clinical focus from the treatment of spasticity to the treatment of sensorimotor dyscoordination in young and older patients with a lesion of the nervous system (see Dietz, this issue).

- What are the limitations of plastic changes in the nervous system? Are such limitations agedependent? Can stem cells help us in partially surpassing these limitation, that is, can they form a mediator of beneficial plastic changes after lesion of the brain?

It will not be easy to get the answers to these questions. It will require joint efforts of clinicians and physiologists. Moreover, the type of physiological research requested is rather a strategy focusing on systems behavior than an approach based on molecular biological techniques. 


\section{REFERENCES}

Anisfeld M. 1991. Review: neonatal imitation. Dev Rev 11: 60-97.

Buonomano DV, Merzenich MM. 1998. Cortical plasticity: From synapses to maps. Ann Rev Neurosci 21: 149-186.

Crothers B, Pain RS. 1988. (originally published in 1959) The Natural History of Cerebral Palsy. London, UK: Mac Keith Press, 270.

Dietz V. 2001. Spinal cord lesion: Effects of and perspectives for treatment. Neural Plast 8: 83-90.

Edelman GM. 1989. Neural Darwinism. The Theory of Neuronal Group Selection. Oxford, UK: Oxford University Press; 371.

Eyre JA, Miller S, Clowry GJ, Conway EA, Watts C. 2000. Functional corticospinal projections are established prenatally in the human foetus permitting involvement in the development of spinal motor centres. Brain 123: 51-64.

Gramsbergen A. 2001. Normal and abnormal development of motor behavior: Lessons from experiments in rats. Neural Plast 8: 17-30.

Hadders-Algra M. 2001. Early brain damage and the development of motor behavior in children: Clues for therapeutical intervention? Neural Plast 8: 3150.

Hepper PG. 1997. Memory in utero? Dev Med Child Neurol 39: 343-346.

Jones SS 1996. Imitation or exploration? Young infants' matching of adult's oral gestures. Child Dev 67: 1952-1969.

Kolb B, Brown R, Witt-Lajeunesse A, Gibb R. 2001. Neural compensations after lesion of the cerebral cortex. Neural Plast 8: 1-16.

Ledoux J. 1996. The emotional brain: The mysterious underpinnings of emotional life. New York, NY, USA: Touchstone; 384.

Lemon RN, Armand J, Olivier E, Edgley SA. 1997. Skilled action and the development of the corticospinal tract in primates. In: Connolly $\mathrm{KJ}$, Forssberg H, eds, Neurophysiology and Neuropsychology of Motor Development. London, UK: MacKeith Press; 78-100.
Martin JH, Kably B, Hacking A. 1999. Activitydependent development of cortical axon terminations. Exp Brain Res 125, 184-199.

Mayston MJ. 2001. People with cerebral palsy: Effects of and perspectives for treatment. Neural Plast 8: 51-70.

Meltzoff AN, Moore MK. 1977. Imitation of facial and manual gestures by human neonates. Science 198: 75-78.

Milner B, Corkin S, Teuber HL. 1968. Further analysis of the hippocampal amnesic syndrome: 14-year follow-up study of H.M. Neuropsychologica 6: 215-234.

Mulder T. Hochstenbach J. 2001. Adaptability and flexibility of the human motor system: Implications for neurological rehabilitation. Neural Plasticity 8 8: $131-140$.

Porter R, Lemon RN. 1993. Corticospinal Function and Voluntary Movement. Oxford, UK: Oxford University Press; 428.

Ramachandran VS, Blakeslee S. 1998. Phantoms of the Brain. London, UK: Fourth Estate; 328.

Rovee-Collier C. 1997. Dissociations in infant memory: Rethinking the development of implicit and explicit memory. Psychol Rev 104: 467-498.

Scrutton D, Baird G. 1997. Surveillance measures of the hips of children with bilateral cerebral palsy. Arch Dis Child 76: 381-384.

Shepherd RB. 2001. Exercise and training to optimize functional motor performance in stroke: Driving neural reorganization? Neural Plasticity 8: 121-130.

Taub E, Miller NE, Novack TA, Cook EW, Fleming WC, Nepomuceno CS, et al. 1993. Technique to improve chronic motor deficit after stroke. Arch Phys Med Rehab 74: 347-354.

Tizard JPM, Paine RS, Crothers B. 1954. Disturbances of sensation in children with hemiplegia. J Am Med Assoc 155: 628-632.

Weindling AM. 2000. Intervention after brain injury to reduce disability. Seminars in Neonatology 5: 53-60.

Wolf SL, Lecraw DE, Barton LA, Jann BB. 1989. Forced use of hemiplegic upper extremities to reverse the effect of learned nonuse among chronic stroke and head-injured patients. Exp Neurol 104: 125-132. 

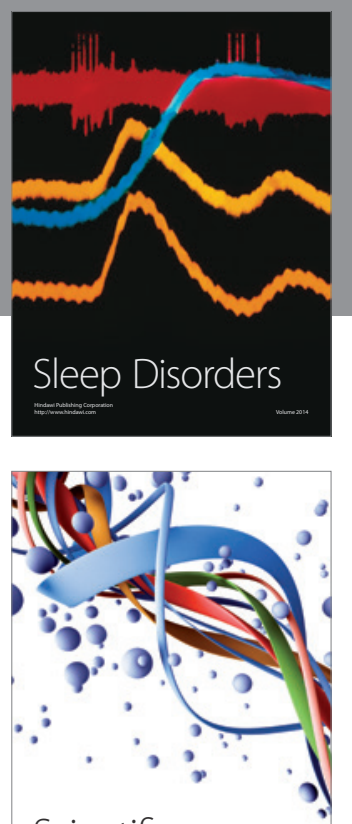

Scientifica
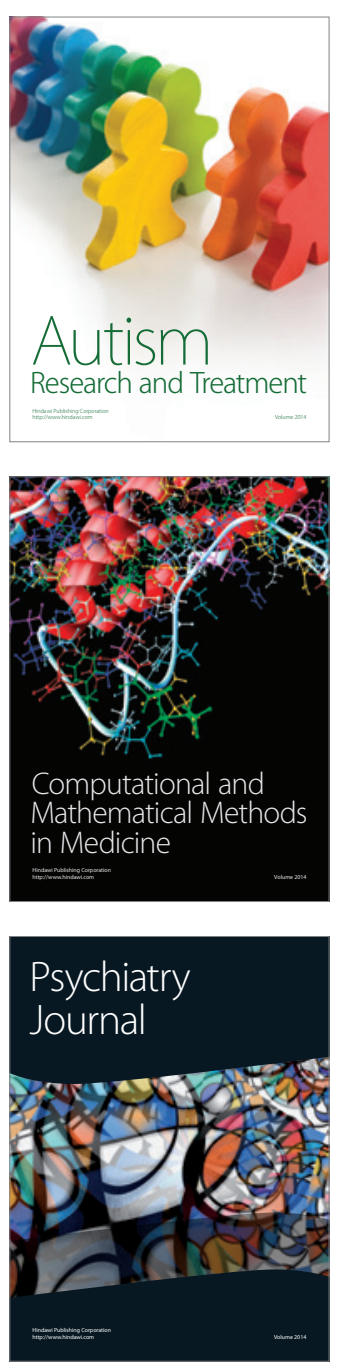
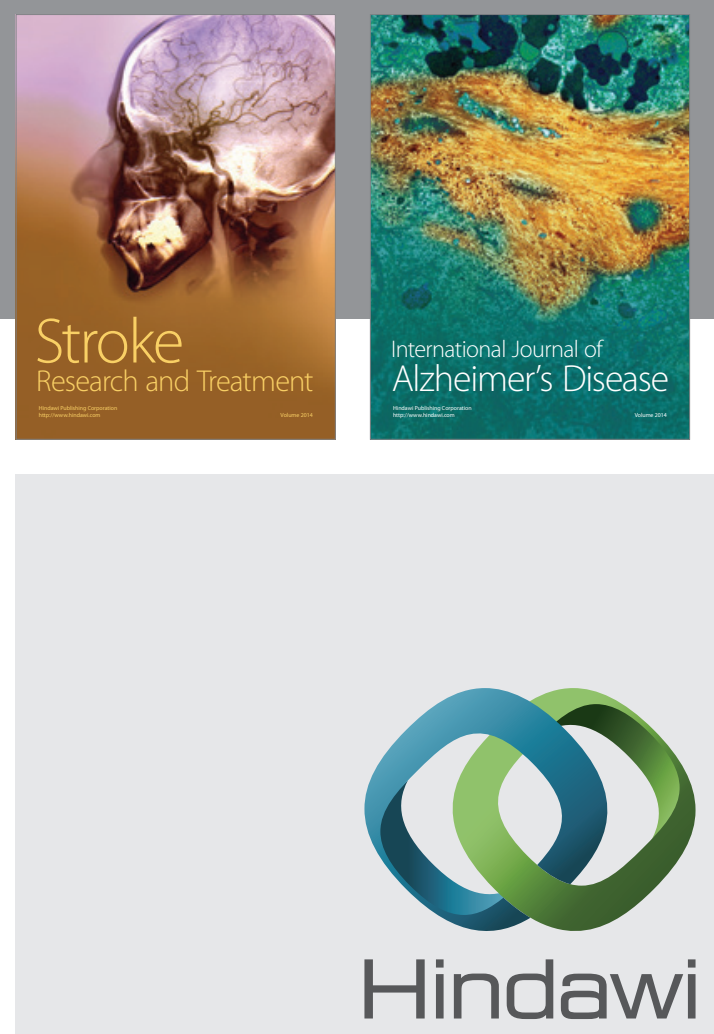

Submit your manuscripts at

http://www.hindawi.com
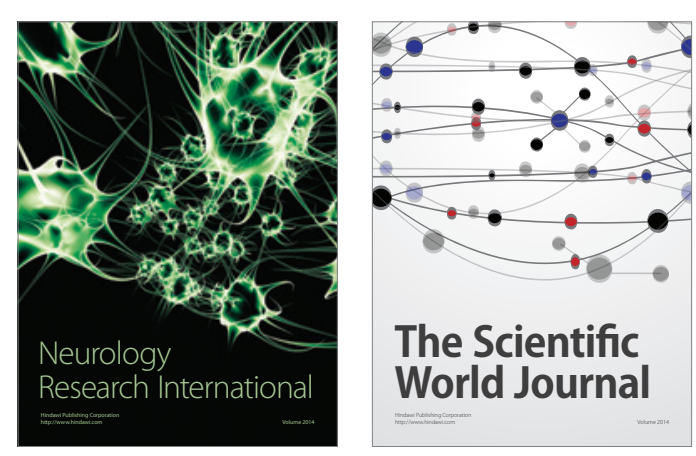

The Scientific World Journal

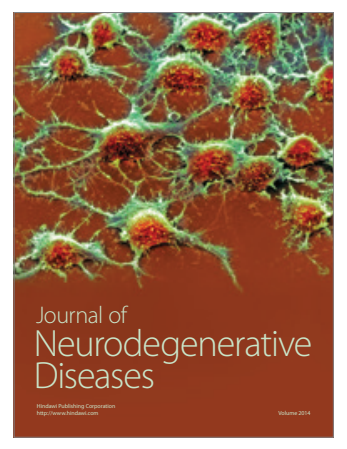

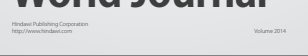

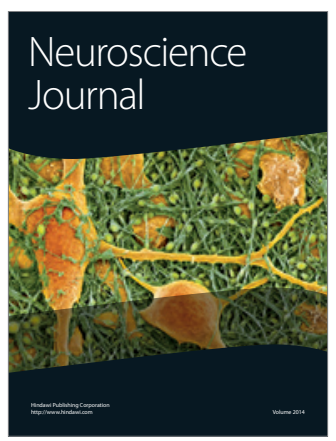

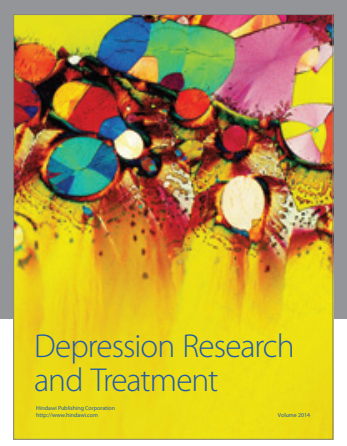
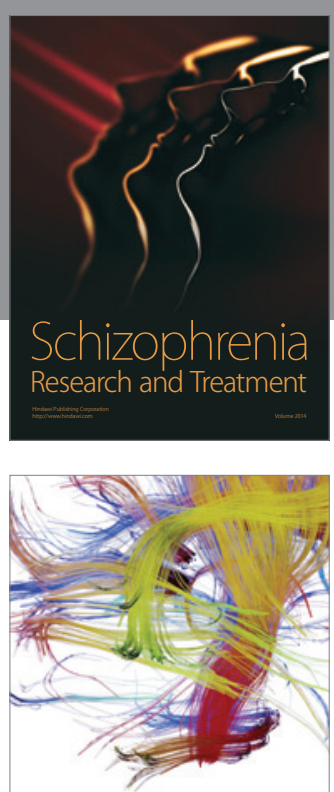

Brain Science

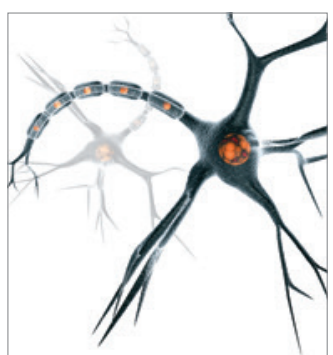

Neural Plasticity
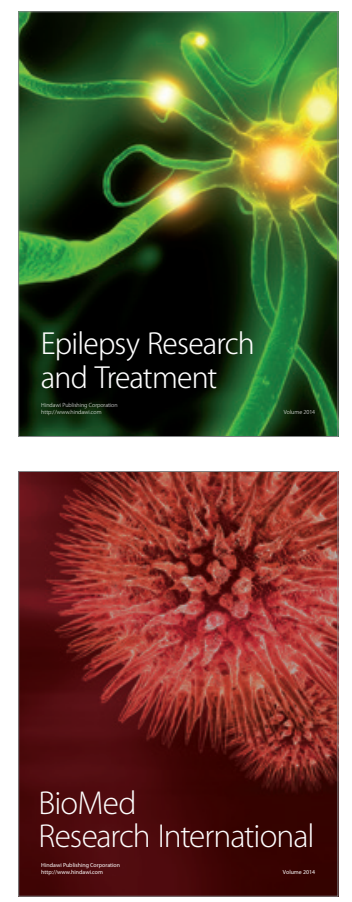

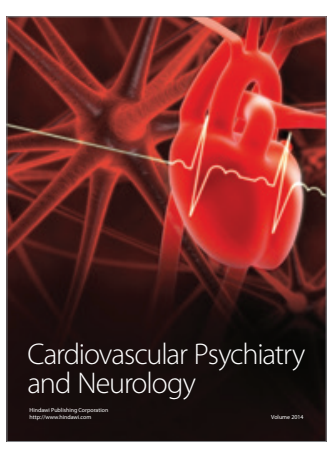

Parkinson's

Disease
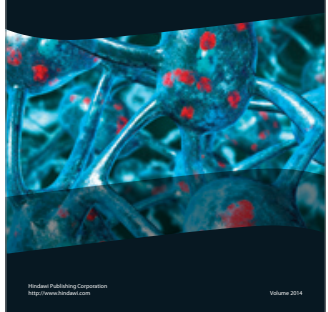